\title{
CARACTERISTICAS DE LA PRUEBA PILOTO: REVISIÓN DE ARTÍCULOS PUBLICADOS EN ENFERMERÍA
}

\author{
CHARACTERISTICS OF THE PILOT \\ TEST: A REVIEW OF ARTICLES \\ PUBLISHED IN NURSING \\ Recibido septiembre 2015 \\ Aceptado noviembre 2015
}

Correspondencia:

Erick Landeros Olvera erick_landeross@hotmail.com nursingresearch@outlook.com

\section{Autores:}

Erika Abeille Mora

Estudiante de la Licenciatura en Enfermería Benemérita Universidad Autónoma de Puebla (BUAP)

Ana Amelia Soto Carrasco

Estudiante de la Licenciatura en Enfermería Benemérita Universidad Autónoma de Puebla (BUAP)

Vivian Paola Muñoz Muñoz

Estudiante de la Licenciatura en Enfermería Benemérita Universidad Autónoma de Puebla (BUAP)

Reina Sánchez Salinas

Estudiante de la Licenciatura en Enfermería Benemérita Universidad Autónoma de Puebla (BUAP)

Saudi Carrera Huerta

Estudiante de la Licenciatura en Enfermería Benemérita Universidad Autónoma de Puebla (BUAP)

Erika Pérez Noriega

Maestra en Ciencias de Enfermería. Miembro del Cuerpo Académico de Enfermería Clínica (BUAP)

Erick Landeros Olvera

Doctor en Ciencias de Enfermería. Miembro del Cuerpo Académico de Enfermería Clínica (BUAP)

Palabras clave: Prueba piloto, validez y conilabilidad

Key words: Pilot-test, validity and reliability 
RESUMEN

Objetivo: Conocer y describir las características de las pruebas piloto que se mencionan en los artículos de investigación en enfermería publicados en un periodo de tres años en un grupo de revistas indizadas.

Metodología: Estudio descriptivo, retrospectivo y transversal. La muestra correspondió a 271 artículos de investigación, fueron seleccionados 42. Se elaboró un instrumento de 18 ítems; sometido al análisis de expertos y piloteado previo a la revisión final para valorar características de los artículos publicados en cuatro revistas Latinoamericanas.

Resultados: El 15.7\% indicaron haber realizado el pilotaje previo y el resto no menciona prueba piloto. Con respecto a las investigaciones que utilizaron procedimientos técnicos, solo el 45\% mencionó capacitación previa de los aplicadores.

Discusión: Identificar previamente que el instrumento es confuso para el sujeto de investigación puede ayudar a que éste pueda ser modificado antes de ser aplicado a una muestra final, y así disminuir el error susceptible.

Conclusión: Sin el reporte de haber realizado previamente una PP, la veracidad y confiabilidad de los instrumentos psicométricos y de los procedimientos técnicos de una investigación disminuyen.

Palabras clave: prueba piloto, validez y confiabililad.

\section{ABSTRAC}

Objective. To know and describe the characteristics of pilot-tests mentioned in nursing research papers published within a three year period in a group of indexed journals.

Method. Descriptive, retrospective and transversal study, with a 271 research articles sample, they were selected 42. An 18-items instrument was elaborated and submitted to experts analysis and then pilot-tested previous to the final review, aiming to evaluate the characteristics of the articles published in four Latin-American journals.

Results. $15.7 \%$ indicated a previous pilot-testing, and the rest did not mention a pilot-test. Regarding researches that used technical procedures, only $45 \%$ mentioned previous training for those in charge of application.

Discussion. Identifying previously that the instrument is confusing for the research subject might help in modifying it before it can be applied on a final sample, decreasing, thus, the susceptible error.

Conclusion. Without reporting the previous PT application, the research psychometric instruments and technical procedures reliability and truthfulness decrease.

Key words: Pilot-test, validity and reliability. 


\section{INTRODUCCIÓN}

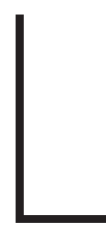

a prueba piloto (PP) es parte de la metodología de investigación que sirve para realizar aproximaciones reales de los proyectos de investigación antes de establecer la prueba final.

Para que un trabajo formalice la recolección de sus datos, debe de tener como antecedente haber realizado una PP; esto contribuye a mejorar la validez y confiabilidad de los procedimientos. Sin embargo, se ha informado que el $69.6 \%$ de los manuscritos originales publicados de enfermería no reportan haber aplicado una PP previa a los datos publicados?. Dicha situación debería considerarse, si la mayoría de las publicaciones con diseños e instrumentos que ameritan la PP, omitieron mencionarla o simplemente no la utilizaron, entonces, es posible que el uso de instrumentos y el entrenamiento de los co-investigadores no fue el adecuado o simplemente no se llevó a cabo; en cualquiera de los casos, para los trabajos de investigación que requieren PP de acuerdo a su diseño y objetivo, es necesario detallar las características con las cuales se llevó a cabo y así contribuir a la garantía de una evidencia de resultados confiable. ${ }^{23,4}$

El objetivo de una PP como simulacro previo a la investigación final, es contribuir a disminuir los posibles sesgos y errores en la obtención de los datos que pueden orientar a mejorar la metodología previamente planteada. Las principales funciones de una PP son: conocer si los procedimientos para la obtención de los datos plateada en la metodología son pertinentes y factibles, conocer la validez previa de los instrumentos y proporcionar entrenamiento a los facilitadores de las intervenciones. Estas características se explican a continuación:

Los procedimientos para la obtención de los datos requieren de la disponibilidad de los recursos necesarios y previamente se requiere aplicarlos en una población similar al de la muestra final o con características ideales para aproximarse a conocer
Ios posibles problemas que pudieran plantearse en la prueba final, así poder realizar las adecuaciones pertinentes para mejorar la factibilidad del estudio.

De la misma forma se prueba la funcionabilidad, veracidad y fiabilidad de los instrumentos, análisis de los datos y la viabilidad del proyecto de investigación 5 .

Independientemente de la consistencia estadística, la validez de los instrumentos depende de varios factores: Por una parte, con la PP se puede evaluar la idoneidad del plan o cuestionario utilizado para la obtención de los datos y determinar la calidad de la tarea del encuestador. Por otra parte, la PP sirve para identificar a través del encuestado o sujeto de investigación si los instrumentos de medición -en el caso de los psicométricos-, fueron largos, cortos, complejos, ambiguos o confusos y de esta forma determinar la factibilidad de aplicación o validez internab. En otras palabras, Ia PP verifica si los sujetos de investigación comprenden el instrumento, si los ítems o preguntas son verdaderamente suficientes y claros, además de que se tiene oportunidad de evaluar la congruencia del lenguaje, redacción o adaptación cultural (si fuera necesario). Estas ventajas de aplicar PP, proporciona al investigador, elementos para realizar algunas modificaciones antes de realizar el muestreo a escala completa?. Sin embargo, la PP no puede ser aplicada a la misma población, es decir, no puede aplicarse en el mismo centro, comunidad, hospital o escuela donde se realizará la recolección final de los datos, no obstante, existen sus excepciones, cuando las poblaciones no son cautivas o previamente conocidas; por ejemplo, cuando se realiza la prueba en pacientes hospitalizados o de una consulta externa que continuamente son rotados o dados de alta.

En el mismo orden de ideas, una de las funciones primordiales de la PP es para dar entrenamiento a los facilitadores de la intervención (colaboradores de la investigación) en los procedimientos técnicos. Para la disciplina de enfermería es muy común obtener indicadores biológicos elementales como 
presión arterial, circunferencia de cintura, obtención de muestras de sangre o alguna otra prueba de clínica o de laboratorio. Este tipo de indicadores, deben de estar sujetas a normas o procedimientos reglamentados que los facilitadores (investigadores) deben de conocer y llevar a cabo con un entrenamiento previo (lo que es parte de una PP).

Durante el entrenamiento a los facilitadores, es necesario que participen todos los colaboradores del proyecto con el objetivo de instruir en el terreno a los diferentes actores que participarán en la selección e inducción de los recolectores de información, la supervisión de la recolección, unificar los procedimientos, recomendaciones técnicas, apoyo logístico ${ }^{8}$ e inclusive realizar un análisis preliminar de los datos de la PP, para agilizar la producción de los informes científicos.

Con respecto a la comunicación que se establece con los sujetos de investigación o la interacción social, la PP otorga elementos para sensibilizar a los que hacen la intervención con respecto al trato y la estandarización de la información que se comunica. Por ejemplo, la forma en solicitar el consentimiento informado o su permiso para poder realizar los procedimientos, ganarse la confianza, garantizar el anonimato de la información personal, aclarar que el protocolo ha sido evaluado previamente por un comité de ética, entre otras cosas. En una PP los investigadores tienen la oportunidad de establecer un vínculo de confianza y poder contestar dudas respecto a los procedimientos durante la aplicación de las pruebas, informar el propósito de estudio, personas o instituciones que lo patrocinan, encargado del proyecto y convocar a reuniones antes y después de las encuestas, para hacer sugerencias y responder inquietudes sobre el instrumento o procedimientos técnicos

Finalmente, uno de los aspectos que más genera polémica: ¿cuántos sujetos se requieren para realizar una PP? De acuerdo García-García, Reding-Bernal y López-Alvarenga?', el tamaño de muestra para estudios piloto se recomienda incluir entre 30 y 50 participantes, los cuales deben poseer los atributos que se desean medir en la población objetivo, esta cantidad sería suficiente para obtener una curva de normalidad en el caso de variables continuas o de razón. Si se tomara en cuenta el número de ítems del instrumento psicométrico, por ejemplo, de 20 ítems, la muestra final podría corresponder a 200 sujetos, la PP se realizaría entre 30 y 60 sujetos que corresponde a un 15-30\%, con lo que se podría correr estadísticos paramétricos.

En otro tipo de diseños, por ejemplo en los ensayos clínicos aleatorios controlados (ECAC), el número de sujetos de la PP puede variar de acuerdo al tamaño de la muestra calculada. En medicina, es común que el ECAC se utiliza para probar acciones terapéuticas o farmacológicas ${ }^{10}$, aquí el tamaño de la muestra se calcula al conocer otros indicadores, por ejemplo la prevalencia de la enfermedad estudiada, ${ }^{11,12}$ sin embargo en una revisión de ensayos clínicos publicados en revistas de alto impacto en un solo año, solo el 33\% justifican el tamaño de la muestra ${ }^{13}$, es posible que los tamaños de muestras sean fijos ${ }^{9}$ y el investigador determina por consecuencia el tamaño de la muestra de la PP sin justificación aparente. Independientemente del método con el que se hava calculado la muestra (coeficiente de relación intraclase, análisis de poder, tamaño de efecto, significancia estadística, población finita) 9.14, la referencia clásica de enfermería de Galvez Toro ${ }^{15}$, indica que en una ECA con un tamaño de efecto pequeño es aquel que oscila entre 11 y 100 sujetos, por lo tanto, podemos considerar que las muestras pilotos podrían aplicarse alrededor de 3 y 30 sujetos de acuerdo a los criterios anteriormente expuestos?.

Sin embargo, debemos puntualizar que no existe una reglamentación al respecto, por lo tanto, debemos aclarar que, aplicar una PP y determinar el tamaño de la muestra, depende del diseño de investigación y del objetivo que se esté buscando, en otras palabras, si el tipo de estudio requiriera de una PP, el número de integrantes para aplicarla dependerá de la metodología ${ }^{16,17}$

\section{2}

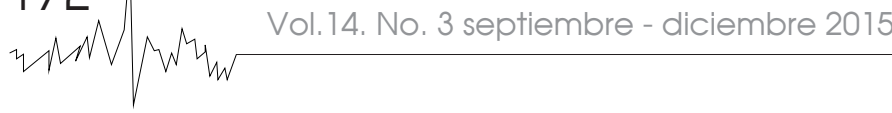


En el caso de las publicaciones científicas de enfermería, han pasado por un proceso de evaluación o arbitraje editorial, el cual dictamina, entre otros aspectos si el método fue adecuado. Si se requiere o no de una PP, lo ideal es que debió reportarse en la metodología, previo a la recolección de final de los datos y detallarse las condiciones y las adecuaciones al método como resultado de la misma. En la literatura, no se ha hecho evidente si en las investigaciones se clarifica las condiciones de la PP y el entrenamiento de los facilitadores de la intervención o encuestadores si es que el diseño lo permitiera. Entonces, bajo esta premisa y con el marco de referencia que establece respecto a las características que debe de tener la PP, surge el siguiente objetivo: Conocer y describir las características de las pruebas piloto que se mencionan en los artículos de investigación en enfermería publicados en un periodo de tres años en un grupo de revistas indizadas.

\section{METODOLOGÍA}

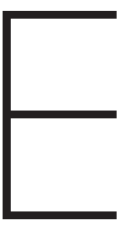

studio descriptivo, retrospectivo y transversal. Se analizaron cuatro revistas latinoamericanas de enfermería indizadas en SciELO. CUIDEN, Lilacs, Scopus-Elsevier. Las revistas seleccionadas fueron: Enfermería Universitaria, Revista de Enfermería del Instituto Mexicano del Seguro Social, Cultura del Cuidado de la Universidad Libre Seccional de Pereira y Aquichan de la Universidad de la Sabana. Las publicaciones corresponden desde el segundo semestre del año 2009 al segundo semestre del 2014. Como criterios de selección de la revista, se consideraron que estuvieran indizadas en base de datos internacionales y reconocidas, con publicación ininterrumpida desde el año 2009. Se consideró solamente la sección de investigación correspondiente de cada revista (artículos originales y artículos de investigación).

La muestra correspondió a 271 artículos de investigación publicados, de los cuales fueron seleccionados 42 artículos, dado que dentro de su diseño se menciona la PP. Se excluyeron aquellos artículos presentados en secciones diferentes (editoriales, sección docencia, práctica profesional, artículos de información académica, clínica y cualitativos, así como procesos de atención de enfermería entre otros).

Antes de proceder al análisis de los artículos, los colaboradores recibieron un entrenamiento en metodología de la investigación de 70 horas. Durante el entrenamiento se elaboró un instrumento para identificar las características de la PP de los artículos, el cual fue sometido al análisis de expertos y previamente piloteado en el análisis de artículos publicados de una revista de circulación nacional, indizada y diferente a las seleccionadas para la conformar la muestra final. El instrumento consta de 18 ítems, los cuales describen las características generales del artículo como las principales temáticas de investigación, marco teórico, diseños, el grado académico e institución de procedencia del primer autor; colaboración con otras disciplinas. Se enfatizó en la sección de metodología, todo lo que pudiera estar redactado haciendo alusión de prueba piloto: validación previa de instrumentos, entrenamientos de encuestadores o facilitadores de intervención, población entre otros aspectos: así como utilización de instrumentos psicométricos. También se consideró identificar la utilización de normas o reglamentos establecidos en el caso de haber usado procedimientos técnicos.

La revisión de los artículos la llevó a cabo un grupo de investigación conformado por estudiantes de Licenciatura en Enfermería generación 2010-2014, bajo la tutela de dos profesores investigadores. Para el análisis de los artículos, se conformaron 3 equipos. Al finalizar la primera revisión, se rotaron los artículos entre los equipos para que fueran objeto de una segunda revisión (cotejo de datos) y de esa manera disminuir el sesgo. El análisis de los resultados se realizó con estadística descriptiva a través del programa SPSS versión 20. Este trabajo fue registrado en la coordinación de investigación de pregrado de Enfermería de la BUAP con el número de registro 099/100. 


\section{RESULTADOS}

L

a producción de artículos publicados por año, a partir del segundo semestre del año 2009 al segundo semestre del 2014 fueron 271 trabajos de investigación. Los resultados respecto a las características generales fueron los siguientes: De los artículos revisados en las secciones de investigación, el 42.1\% son de carácter clínico, el 19.8\% son de educación para la salud y el resto son de temáticas de enfermería familiar, comunitaria, administración y otros.

Los resultados respecto a las características de los artículos que reportaron PP fueron los siguientes: El 15.7\% (42 de 271 artículos), indicaron haber realizado PP. De éstos que la mencionan, el 74\% (31 de 42), indicaron haber piloteado instrumentos psicométricos. Sin embargo, 55\% (23 de 42) de los estudios que reportaron PP del instrumento psicométrico y que además requirieron utilizar procedimientos técnicos (datos clínicos, antropometría y obtención de muestras sanguíneas), no es claro si se estandarizaron bajo una norma o reglamentación con la cual se haya capacitado a los investigadores.

Se identificó que el 93\% (39 de 42 artículos) que realizaron PP, no consideraron cambios metodológicos en los procedimientos para la recolección final de los datos. Del total de artículos que mencionaron haber realizado PP, el 86\% (36 de 42) no mencionó en qué tipo de población se realizó, no es claro si la PP se realizó en una muestra con características similares a la muestra final o en el mismo lugar de la prueba final. Finalmente, el 48\% (20 de 42) no mencionó el número de sujetos que conformaron la muestra piloto.

\section{DISCUSIÓN}

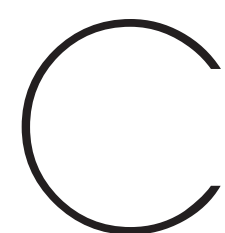

uando un instrumento psicométrico es confuso para el sujeto de investigación puede deberse a la redacción de las preguntas o afirmaciones, o bien no es adecuado al contexto sociocultural educativo de éste. Identificar previamente esta situación puede ayudar a que los instrumentos puedan ser modificados antes de ser aplicados a una muestra final, así, disminuir el error susceptible y el error tipo III, que se derivan de fallos en la intervención o aplicación de los instrumentos, e inclusive del análisis estadístico 18,19.

El presente trabajo de investigación pretendió dar a conocer y describir la aplicación de la PP que se mencionan en los artículos de investigación publicados en las revistas de enfermería en un periodo de tres años. Se analizaron 271 artículos en la sección de investigación de 4 principales revistas de enfermería Latinoamericanas, de los cuales fueron 42 artículos que mencionaron aplicar la PP. Los principales hallazgos en las características generales fueron las siguientes:

a) Los temas clínicos y de educción para la salud son los que más se publican.

Estos hallazgos son congruentes con el reporte de García et al.?', quienes indicaron que los temas de investigación más publicados en enfermería son los clínicos, lo cual se relaciona ampliamente con los resultados encontrados en nuestra investigación, la tendencia no es diferente.

b) Gran parte de los estudios analizados no mostraron haber realizado pilotaje previo de los instrumentos psicométricos empleados, al parecer se da por hecho que no es necesario, pues se encuentran referencias de la validación previa de dichos instrumentos, sin considerar que existen otros factores que pueden disminuir la validez de un instrumento y no solo desde el punto de vista estadístico.

c) De los artículos analizados; gran parte de los que utilizaron procedimientos técnicos, no mencionan el procedimiento para la estandarización - la normatividad que los guió para recolectar los datos correspondientes. Es posible que la preparación se tenga de formación o sí se haya considerado estandarizar los procedimientos, pero al no mencionarlos queda poco claro la forma en que se procedió para la recolección de los datos clínicos, de laboratorio y antropométricos principalmente.

\section{4}

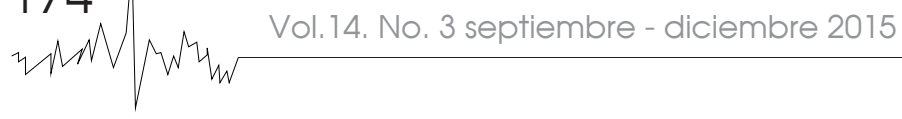


d) De los artículos que realizaron PP, un escaso porcentaje requirió considerar cambios metodológicos en los procedimientos, situación que apoya para mejorar o garantizar la recolección de los datos final y contribuir a disminuir el sesgo.

e) Con base en los resultados encontrados, nos permite identificar que la PP -al parecer-, no fue aplicada a una muestra con características similares a la muestra final, esto no es concluyente, pues el dato se pudo omitir en la publicación, pero es posible que las investigaciones se hayan editado a pesar de los sesgos.

f) De los artículos que mencionan la utilización de la PP, solo 20 señalan cuántos sujetos formaron parte de dicha prueba. No clarifican el proceso para decidir el tamaño de la muestra piloto, situación que era de esperarse dado que la revisión de la literatura en estudios similares, indican que no se redacta la justificación del cálculo de la muestra ${ }^{13}$.

\section{CONCLUSIONES}

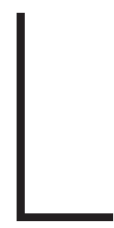

os artículos que de acuerdo a su diseño requirieron de una prueba piloto, no expresan claramente las condiciones de su aplicación, entrenamiento, tamaño de muestra y población seleccionada; posiblemente omiten mencionarla. El reporte de estos hallazgos no es concluyente, pero representa una aproximación a los reportes de la enfermería latinoamericana, donde se puede observar que aún existen deficiencias que indican un pobre rigor metodológico en los trabajos de investigación publicados.

\section{AGRADECIMIENTO}

A las seis alumnas del quinto cuatrimestre la sección 01 de la Licenciatura de la FE-BUAP generación 2010-2014, quienes por consenso aleatorio y consentimiento firmado no están como co-autores de esta publicación. Carreón-Muñoz María Fernanda, López Cuellar Irma, Martínez Sánchez Johana Beatriz, Padilla Pérez Lourdes Margarita, Rodríguez González Laura y Zaragoza Sánchez Gabriela.

\section{REFERENCIAS BIBLIOGRÁFICAS}

1. García-Rodríguez M, Gómez Ávila M, Aguilar-Pérez I, Pérez-Martínez G, Velázquez-Díaz L, Soriano Sotomayor M, Landeros-Olvera E. Tendencias y características de la investigación en enfermería publicada en México. Rev Enf Uni. 2011; 8(1): 7-16.

2 National Library of Medicine. Citing medicine: the NLM Style Guide for Authors, Editors, and Publishers (Internet). 2nd ed. 2007. (Citado enero 2016). Disponible en: http://www.ncbi.n/m.nih.gov/books/NBK7256/?depth=2

3. Foster RL. Preferred reporting items for systematic reviews and metaanalyses. J Spec Ped Nur. 201 1; 17:1 fi2.

4. Cobos-Carbó A, Augustovski F. Declaración CONSORT 2010: actualización de la lista de comprobación para informar ensayos clínicos aleatorizados de grupos paralelos. Med Clin. 2011;137(5):213fi215.

5. Polit D, Tatano B. Nursing Research. Generating and Assessing Evidence for Nursing Practice. 9th Ed. Philadelphia: Lippincott, Williams and Wilkins source publications; 2012. 236 p.

6. Muñiz J, Elisva P, Hambleton R. Directrices para la traducción y adaptación de los test. Psico. 2013; 25(2): 151-157

7. García G, Jiménez P, Arnaud V, Ramírez T, Lino P. Introducción a la metodología de investigación en ciencias de la salud. Tra Ed. México: McGraw-Hill; 2011.50 p.

8. Ordoñez NS, Bustamante GM, Anzícar JG. Programa de apoyo a la reforma de salud crédito bid 910/0c-co (Internet). Colombia: informe de los hallazgos Técnicos en la Prueba Piloto. (Citado en enero 2016). Disponible en: https://www.minsalud.gov.co/salud/Documents/ Informe\%2Ofinal\%20piloto.pdf

9. García-García JA, Reding-Bernal A, López-Alvarenga JC. Cálculo del tamaño de la muestra en investigación en educación médica. Inv Ed Med. 2013; 2(8):217-224.

10. Llor C, Moragas A. Dil̈cultades de selección en los ensayos clínicos en atención primaria. Cuando no se llega al tamaño muestral. Aten Primaria. 2013:45(2):71-73

11. M. Miravitlles, J.J. Soler-Cataluña, M. Calle, J. Molina, P. Almagro, J.A. Quintano, et al. Spanish COPD guidelines (GesEPOC). Pharmacological treatment of stable COPD. Arch Bronconeumol. 2012; 48: 247 fi257

12. Vanegas ID, Zuluaga GM, Donado Gómez JH. Diferencias entre las características basales de los pacientes en los ensayos clínicos, según el tamaño de la muestra. Un estudio metaepidemiológico. Medicina u.p.b. 2013; 32(1): 30-36

13. Silva AL, Gallbánb PA. Explicación del tamaño muestral empleado: una exigencia irracional de las revistas biomédicas. Gac Sanit. 2013:27(1):53fi57

14. Piaggio $G$, Elbourne DR, Stuart JP, Stephen JW, Douglas GA. Reporting of Noninferiority and Equivalence Randomized Trials Extension of the CONSORT 2010 Statement. JAMA. 2012;308(24):2594-2604

15. Gálvez Toro A. Enfermería Basada en la Evidencia. Cómo incorporar la Investigación a la Práctica de los Cuidados. Fundación Index. 2" Ed. Granada; 2007. 179 p.

16. Grove, Burns, Gray. The practice of nursing research. Appraisal, synthesis, and generation of evidence. 7th ed. St. Louis, Missouri: Elsevier Saunders; $2013.523 \mathrm{p}$.

17. Hernández SR, Fernández CC, Baptista LP. Metodología de la Investigación. 6th Ed. México: McGraw-Hill/ Interamericana; 2014. 415 p.

18. Mackey A, Gass SM. Second Lenguaje Research, Methodology and Design. 2th Ed. New York and London: Routledge; 2016.52 p.

19. Sidani S, Braden CJ. Design, Evaluation, and Translation of Nursing Interventions. 1th. lowa: Wilwy-Blacwell; 2011.56 p. 